\title{
ARMADILHAS FOTOGRÁFICAS COMO MÉTODO DE REGISTRO DE MAMÍFEROS DE MÉDIO E GRANDE PORTE NA SERRA DA JIBOIA, BAHIA
}

Bianca de Moura Calixto ${ }^{\mathbf{1}}$; Téo Veiga de Oliveira ${ }^{2}$;

1. Bolsista PIBIC/Fapesb, Graduando em Ciências Biológicas, Universidade Estadual de Feira de Santana, e-mail: ban_calixtof2@hotmail.com

2. Orientador, Departamento de Ciências Biológicas, Universidade Estadual de Feira de Santana, e-mail: teovoli@yahoo.com.br

PALAVRAS-CHAVE: mamíferos; armadilhas fotográficas; Mata Atlântica.

\section{INTRODUÇÃO}

Mamíferos terrestres de médio e grande porte desempenham um importante papel em vários níveis da organização de um ecossistema, podendo ser destacados o controle populacional de suas presas, no caso das espécies carnívoras, e a constante regeneração das matas, no caso dos herbívoros (Emmons \& Feer, 2005; AbreuJr. \& Kohler, 2009; Sinclair, 2003; Tonhasca Jr., 2005); mamíferos maiores são importantes também por agruparem diversas espécies consideradas indicadoras ambientais, refletindo a preservação do local onde ocorrem (Mazzolli, 2006).

A presença e a abundância destes mamíferos em determinada localidade é afetada não só pela qualidade ambiental, mas também pelo fato de serem os principais alvos de atividades humanas como a caça ilegal (Cuarón, 2000). Apesar disto, mamíferos terrestres de médio e grande porte são pouco estudados devido às dificuldades referentes ao seu registro; em muitos inventários as armadilhas utilizadas não permitem a captura de mamíferos maiores ou, mesmo que sejam utilizadas armadilhas grandes, o menor tamanho das populações destes mamíferos dificulta sua captura.

A instalação de armadilhas fotográficas na área estudada auxilia na obtenção de dados, uma vez que, é possível registrar mamíferos de médio e grande porte sem necessidade de capturá-los e consequentemente, causar perturbações e stress nos animais.

Desta forma, este plano de trabalho oferecerá informações adicionais ao inventário em relação às espécies registradas através da captura em armadilhas, sendo uma ferramenta complementar importante ao conhecimento mais completo das mastofaunas da Mata Atlântica baiana, especificamente na Serra da Jiboia.

\section{MATERIAL E MÉTODOS}

As expedições de campo foram desenvolvidas na Serra da Jiboia, que abrange cinco municípios: Varzedo, Elísio Medrado, Santa Terezinha e Castro Alves, no Recôncavo Baiano.

Foram instaladas três armadilhas fotográficas, sendo duas configuradas para registrar através de fotos e uma por vídeos, em pontos da mata que sugeriam o trânsito de mamíferos (próximos a cursos d'água, com sinais de trilhas produzidas por animais).

No chão, à frente das armadilhas, foram jogados pedaços de mandioca, milho verde e batata doce, também com fins atrativos.

As armadilhas permaneceram ligadas ininterruptamente desde o início da campanha até o mês seguinte, quando serão removidas e trocadas de lugar (O'Connel et al., 2011; Glen etal., 2013).

\section{RESULTADOS E DISCUSSÃO}


Foram realizadas três expedições de campo para a região de Pancada, em Varzedo-BA. As armadilhas permaneceram em campo de Dezembro de 2017 até Junho de 2018. Em Fevereiro de 2018 foram revisadas e foram coletados os dados do período para análise, o mesmo em Junho de 2018, juntamente com a remoção das armadilhas do campo.

No total foram registradas 10 espécies (Tabela 1), distribuídas nas ordens Didelphimorphia, Carnivora, Cetartiodactyla, Cingulata, Pilosa e Rodentia. Sendo as espécies registradas Cuniculus paca (Fig 1 - g), Dasyprocta prymnolopha (Fig 1 - a), Dasypus sp., Didelphis sp.(Fig 1 - d), Eira barbara (Fig 1 - e), Leopardus pardalis (Fig 1 - f), Nasua nasua (Fig 1 - b), Pecari tajacu (Fig 1 - c), Procyon cancrivorus, Tamandua tetradactyla (Fig 1 - h), além de muitos registros de cão doméstico, algumas aves e teiú. As espécies que não se encontram na figura não foram adicionadas porque os registros das mesmas não foram satisfatórios ou não estão bem visíveis para vir em anexo. Neste trabalho, obteve-se um considerável número de registros e espécies, mesmo com a presença de cães domésticos que poderiam afugentá-las. Este fato pode ser atribuído ao tempo em que essas armadilhas permaneceram em campo. O destaque para o registro da Irara (Eira barbara) que até então não havia sido registrada nesta localidade da Serra.

Logo, o uso de armadilhas fotográficas mostrou-se um método eficaz para a obtenção de dados sobre a mastofauna, sobretudo da Serra da Jiboia, pois até este presente trabalho registrou-se espécies comumente encontradas, além de registros de espécies que nunca haviam sido registradas antes nesta localidade.

Tabela 1. Lista de espécies registradas na Serra da Jiboia, localidade de Pancada, Varzedo-BA.

\title{
Espécies
}

\author{
Ordem Didelphimorphia \\ Didelphis sp \\ Ordem Carnivora \\ Eira barbara \\ Leopardus pardalis \\ Nasua nasua \\ Procyon cancrivorus \\ Ordem Cetartiodactyla \\ Pecari tajacu \\ Ordem Cingulata \\ Dasypus sp. \\ Ordem Pilosa \\ Tamandua tetradactyla \\ Ordem Rodentia \\ Cuniculus paca \\ Dasyprocta prymnolopha \\ Legenda: 1. Espécies separadas pela ordem a qual pertencem.
}




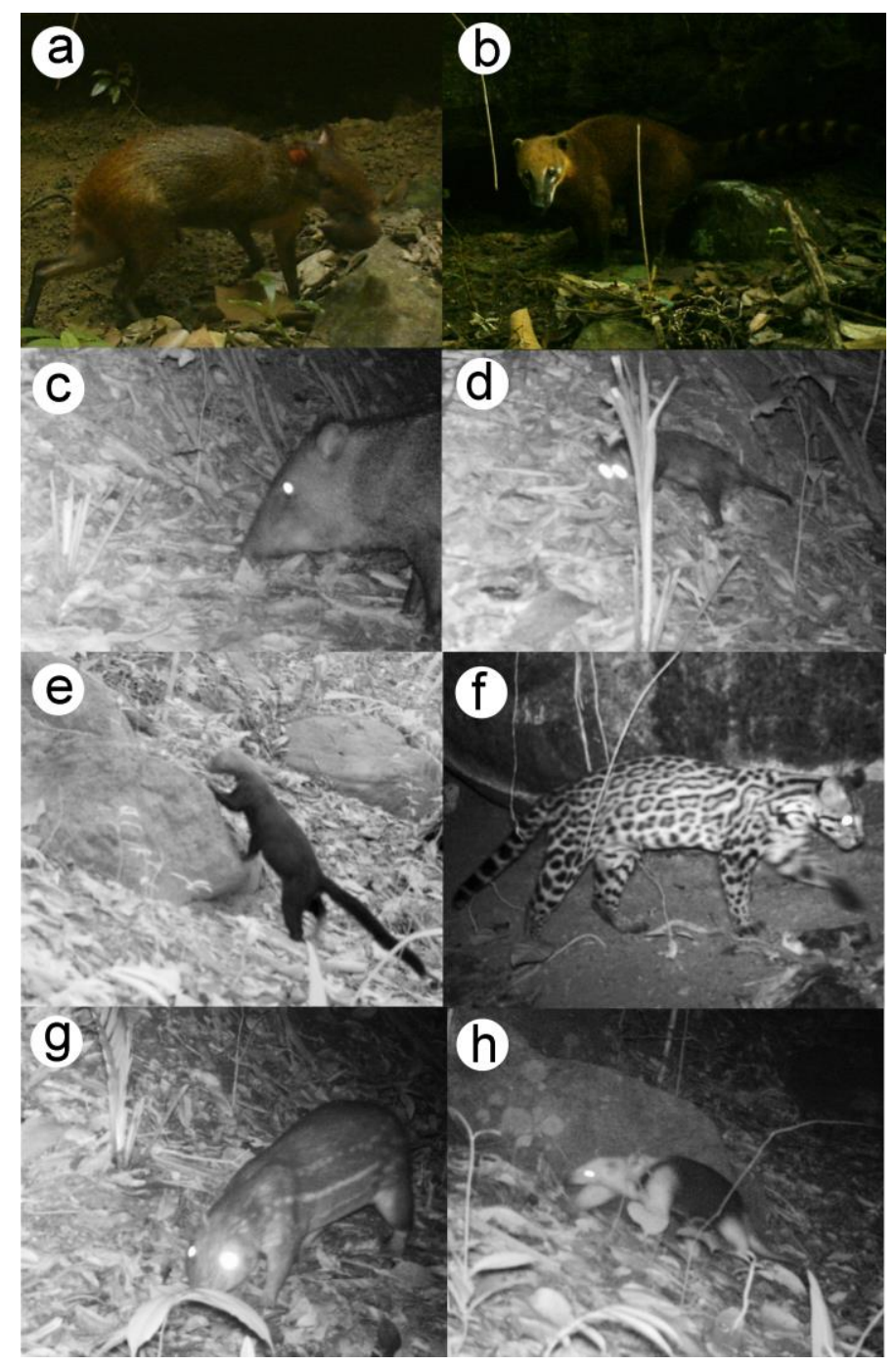

Figura 1: Algumas das espécies registradas através das armadilhas fotográficas. a - Dasyprocta prymnolopha; b - Nasua nasua; c Pecari tajacu; d - Didelphis sp.; e - Eira bárbara; f - Leopardus pardalis; g-Cuniculus paca; $\mathrm{h}$ - Tamandua tetradactyla.

\section{CONSIDERAÇÕES FINAIS}

$\mathrm{O}$ uso das armadilhas fotográficas veio a contribuir para um inventário mais completo da Serra da Jiboia, já que, registra a presença de espécies que não aparecem através de outros métodos de amostragem e seu uso na Serra possibilitou a amostragem de espécies que, até então, não haviam sido registradas antes.

\section{REFERÊNCIAS}


ABREU JR., E.F. \& KÖHLER, A. 2009. Mammalian fauna of medium and large sized in the RPPN of UNISC, RS, Brazil. Biota Neotropica, vol. 9, no. 4.

CUARÓN, A.D. 2000. A global perspective on habitat disturbance and tropical rainforest mammals. Conservation Biology, v.14, n.6, p.1574-1579.

EMMONS, L. \& FEER, F. 1997. Neotropical rainforest mammals. A field guide. Second edition. Chicago: The University of Chicago Press 307p.

GLEN, A.S.; COCKBURN, S.; NICHOLS, M.; EKANAYAKE, J. \& WARBURTON, B. 2013. Optimising camera traps for monitoring small mammals.P losOne, 8:1-7.

MAZZOLLI, M. 2006. Persistência e riqueza de mamíferos focais em sistemas agropecuários no planalto meridional brasileiro. Tese de Doutorado, Universidade Federal do Rio Grande do Sul, Porto Alegre.

O'CONNELL; A.F., NICHOLS; J.D. \& KARANTH, K.U. 2011. Camera traps in animal ecology: methods and analyses. New York: Springer, 271p.

SINCLAIR, A. R. E. 2003. Mammal Population Regulation, Keystone Processes and Ecosystem Dynamics. Philosophical Transactions: Biological Sciences, 358(1438): 1729-1740.

TONHASCA JR., A. 2005. Ecologia e história natural da Mata Atlântica. Interciência, Rio de Janeiro, 197 p. 